\title{
Estimación en cálculo multiplicativo con números decimales
}

\section{Multiplicative computational estimation with decimal numbers}

\author{
Carlos de Castro Hernández \\ Universidad Autónoma de Madrid \\ carlos.decastro@uam.es \\ Enrique Castro Martínez, Isidoro Segovia Álex \\ Universidad de Granada \\ ecastro@ugr.es, isegovia@ugr.es
}

RESUMEN • La estimación en cálculo es una destreza básica, útil en la resolución de problemas aritméticos. En este artículo analizamos las dificultades, estrategias y errores en las estimaciones de cálculos aritméticos de multiplicación y división con números naturales y decimales. Empleamos una metodología mixta, cuantitativa-cualitativa, recogiendo datos mediante una prueba de estimación, seguida por una fase de entrevistas. Participan 131 estudiantes de Magisterio. Entre los resultados, los ítems con decimales menores que 0,1 son los más difíciles; con decimales menores que 1 , más difíciles que con naturales o decimales mayores que 1; y las divisiones $\mathrm{B}$ (dividendo menor que divisor), más difíciles que las divisiones A (dividendo mayor que divisor). En la parte cualitativa, con entrevista semiestructurada, analizamos las estrategias y los errores al estimar. Detectamos cuatro estrategias básicas (primeros dígitos, sustitución, fracciones y algoritmo alternativo) y 15 tipos de errores (4 en la interpretación, 10 en la ejecución y 1 en la evaluación).

PALABRAS CLAVE: estimación en cálculo; estructura multiplicativa; números decimales; estrategias; errores.

SUMMARY • Computational estimation is a basic skill, useful in solving arithmetic problems. In this paper, we analyze the difficulties, strategies and errors in computational estimation of multiplications and divisions with natural and decimal numbers. We use a mixed methodology, quantitative and qualitative, collecting data by means of an estimation test, followed by a phase of interviews. Participants in the study were 131 pre-service teachers. Among the results, tasks with decimals less than 0.1 are more difficult than others; tasks with decimals less than 1, more difficult than those with natural or decimals greater than 1 ; and $\mathrm{B}$ divisions (with dividend less than divisor) are more difficult than A divisions (with dividend larger than divisor). Qualitative study shows 3 basic estimation strategies (First digits, fractions, and alternative algorithm) and 15 types of errors ( 4 in interpretation phase, 10 in computation phase, and 1 in evaluation).

KEYWORDS: computational estimation; multiplicative structure; decimal numbers; strategies; errors.

Fecha de recepción: septiembre 2012 • Aceptado: febrero 2013

De Castro Hernández, C., Castro Martínez, E., Segovia, I. (2014) Estimación en cálculo

multiplicativo con números decimales. Enseñanza de las Ciencias, 32 (2), pp. 171-190 


\section{INTRODUCCIÓN}

La estimación ha sido reconocida como una destreza útil, valorada en una amplia variedad de profesiones y situaciones de la vida real. La rapidez y potencia de las calculadoras y ordenadores actuales hacen imprescindible que las personas sean capaces de detectar la razonabilidad de los cálculos obtenidos mediante esta tecnología, ya presente en el currículo escolar. Los profesores de matemáticas tienen que ser conscientes de ello y poseer una formación adecuada que les permita, por un lado, juzgar las propuestas curriculares al respecto y, por otro, programar actividades adecuadas al nivel de los escolares. Por ello, los programas de formación de los profesores deben dar soporte para que estos adquieran la competencia necesaria que les permita formar a los escolares como buenos estimadores, lo que conlleva que adquieran habilidades de estimación y confianza en estas.

La estimación la definimos como un "juicio sobre el valor del resultado de una operación numérica o de la medida de una cantidad, en función de circunstancias individuales del que lo emite" (Segovia, Castro, Rico y Castro, 1989: 18). Dentro del ámbito de la estimación distinguimos la estimación en cálculo, la de la numerosidad de cantidades discretas y la de la medida de cantidades continuas (Hogan y Brezinski, 2003; Segovia y De Castro, 2007; Segovia y otros, 1989). En este trabajo, nos centramos en la estimación en cálculo, definida como "el proceso de transformar números exactos en aproximaciones y calcular mentalmente con estos números para obtener una respuesta razonablemente próxima al resultado exacto de un cálculo" (Sowder, 1988: 182). Esta definición enfatiza tres aspectos característicos de la estimación: las destrezas de aproximación (como el redondeo o el truncamiento), el vínculo existente entre estimación y cálculo mental y el concepto de razonabilidad del resultado, claves para comprender la estimación. Dentro de la estimación en cálculo, nuestro trabajo va a estar referido a las operaciones de multiplicación y división con números naturales y decimales.

La estimación se introduce en el currículo español de Educación Primaria en 1991: "Las matemáticas constituyen hoy un conjunto amplio de modelos y procedimientos de análisis, de cálculo, medida y estimación” (MEC, 1991, p. 31). Previamente, en 1986, el National Council of Teachers of Mathematics (NCTM) dedica el Yearbook de ese año a la estimación, dado el cada vez mayor reconocimiento por los profesores de matemáticas de la importancia de la enseñanza y aprendizaje de la estimación (Schoen y Zweng, 1986, p. vii); poco tiempo después, en 1987 se redacta el primer borrador de "Estándares curriculares y de evaluación para la Educación Matemática”, editado en España en 1991, en el que el estándar número 5 está dedicado a la estimación (NCTM, 1991). Más recientemente, en los "Principios y estándares para la Educación Matemática” (NCTM, 2003), se plantea la necesidad de desarrollar programas de enseñanza que permitan a los alumnos calcular con fluidez, hacer estimaciones razonables y juzgar la razonabilidad de resultados obtenidos por otros medios de cálculo. La estimación se considera parte fundamental del sentido numérico, definido como red conceptual de naturaleza intuitiva que permite emitir juicios cuantitativos, comparar números, valorar la razonabilidad de resultados de cálculos, emplear formas no algorítmicas de cálculo mental, o resolver problemas con flexibilidad (Sowder, 1988).

A partir de estos documentos, la presencia y relevancia de la estimación en los currículos de matemáticas son crecientes. En España, el currículo de Educación Primaria propone, como objetivo general: "Desarrollar las competencias matemáticas básicas e iniciarse en la resolución de problemas que requieran la realización de operaciones elementales de cálculo, conocimientos geométricos y estimaciones, así como ser capaces de aplicarlos a las situaciones de su vida cotidiana” (MEC, 2007: 31488). Dentro del bloque de números y operaciones, se pretende "esencialmente el desarrollo del sentido numérico" (p. 31555) y que los niños calculen "con fluidez y hagan estimaciones razonables" (p. 31556). La propia idea de competencia básica, con carácter transversal a los contenidos, y enfatizando la aplicación de los conocimientos, facilita la incorporación, cada vez más decidida, de la estimación en el currículo mate- 
mático. En Estados Unidos, dos documentos protagonizan la línea de desarrollo del currículo de matemáticas: los "Curriculum focal points" (NCTM, 2006), donde la estimación aparece como contenido fundamental en todos los cursos, y los "Common core standards" (NGA Center \& cCsso, 2010), donde la estimación aparece ligada a la notación exponencial y a la expresión aproximada de grandes cantidades. En este documento también se propone estimar la solución de ecuaciones a través de sus representaciones gráficas, la tasa de variación de una función y la aplicación de la estimación a la modelización.

El currículo matemático de Educación Primaria, con el énfasis mostrado en el sentido numérico y la estimación, debe ser desarrollado en última instancia por profesores en el aula. La formación inicial de estos debe incluir especial atención a la estimación y al sentido numérico (Fung y Latulippe, 2010). Pero esta necesidad choca con la dificultad encontrada en investigaciones para introducir la estimación en el currículo, como en Alajmi (2009), donde dos tercios de los profesores kuwaitíes de matemáticas percibían la estimación como una destreza importante para desenvolverse en la vida diaria, aunque solo a un $20 \%$ les parecía relevante para la educación matemática, y más del $50 \%$ se mostraban poco dispuestos a enseñarla. Edwards (1984) daba algunas razones por las que la estimación no llegaba a incorporarse de la manera más adecuada al currículo: por la multiplicidad de métodos, por el carácter de las respuestas que no podían considerarse buenas o malas, por la dificultad de la evaluación y porque para los estudiantes, y quizás también para los profesores, la estimación es vista como algo inferior en matemáticas.

\section{Marco teórico para la estimación de cálculos multiplicativos}

Tras justificar el interés de investigar la estimación con profesores, y debido a la amplitud del campo de la estimación, nos centramos en la estimación en cálculos multiplicativos, siguiendo ideas presentes en revisiones de estudios sobre problemas de estructura multiplicativa (Castro y Castro, 1996; De Corte y Verschaffel, 1996). Según estas revisiones, los niños tienen dificultades para elegir la operación adecuada en problemas de estructura multiplicativa con números decimales menores que uno. Estas dificultades se deben a las ideas equivocadas sobre el efecto de multiplicar o dividir por decimales menores que uno ("la multiplicación aumenta", "la división disminuye" y "siempre se divide un número por otro menor”), ideas válidas con números enteros, que fallan al extrapolarse a los decimales menores que la unidad, y que tendrían su origen en el predominio en la enseñanza del modelo de adición repetida para la multiplicación y el modelo de reparto para la división (De Corte y otros, 1996). Por otra parte, Castro y Castro (1996: 124-126), al revisar estudios de los ańos ochenta y noventa, sobre el conocimiento de profesores en formación sobre la multiplicación y la división, advierten que los propios modelos de la multiplicación y la división también generan dificultades en los profesores. Además, los profesores tienen dificultades con la interpretación de la división como medida, lo que afecta a la división de fracciones, y deficiencias en el conocimiento de la división, con lagunas en el conocimiento conceptual, y en la interpretación del resto o la parte fraccionaria del cociente.

Ya en el ámbito de la estimación, se ha encontrado que estimar resultados de multiplicaciones y divisiones con decimales (mayores y menores que 1) es más difícil que hacerlo con números naturales (Bestgen, Reys, Rybolt y Wyatt, 1980; De Castro, 2002; Rubenstein, 1985). Sin embargo, si se toman solo decimales mayores que uno, no se encuentra diferencia significativa de dificultad entre las tareas con números naturales y decimales (Goodman, 1991). Profundizando en este sentido, Cortés, Backhoff y Organista (2005) encuentran dificultades en estudiantes de secundaria para estimar con decimales menores que uno ( $486 \times 0,24$ tuvo un $33 \%$ de respuestas no razonables). Igualmente, Volkova (2006) indica que hay profesores que piensan que la estimación del producto de fracciones debe ser mayor que ambos factores, y lo aplican al producto $3 \frac{1}{2} \times 9 / 10$ para dar una estimación de 4 . Ante la división $11 / 12 \div 3 / 4$, algunos profesores razonaron que el resultado debía ser menor que 1 , pues ambas fracciones lo eran. Yang, Reys y Reys (2009) concluyen, en su trabajo sobre sentido numérico, tras in- 
vestigaciones realizadas con estudiantes y profesores de Taiwán, Kuwait, Suecia, Japón y Australia, que los participantes, al trabajar con números decimales, tienen un conocimiento pobre sobre el efecto de la alteración de los datos en el resultado de una operación (como, por ejemplo, multiplicar un número por otro menor que 1 ). Dentro de los decimales menores que 1 , los decimales menores que 0,1 parecen presentar dificultades mayores que los que están entre 0,1 y 1 (De Castro, 2002; Levine, 1980).

Recientes revisiones de estudios sobre estimación (Segovia y Castro, 2009; Segovia y De Castro, 2007, y Siegler y Booth, 2005) coinciden en señalar la investigación en estrategias de estimación como uno de los campos de mayor interés dentro de la estimación. Esto es debido, en parte, a que los procesos y estrategias de estimación difieren en gran medida de los algoritmos de cálculo tradicionales. Estos son procedimientos más rígidos, frente a la flexibilidad que se intenta promover a través de las estrategias de estimación (Star y Rittle-Johnson, 2009; Star, Rittle-Johnson, Lynch y Perova, 2009). Dentro de las estrategias, Siegler y Booth (2005), en la estimación, y Gómez (1995a), en cálculo mental, destacan que las estrategias se pueden estudiar en diferentes niveles de generalidad. En un primer nivel, se pueden estudiar los procesos de estimación, descritos en el modelo RTC de Reys, Bestgen, Rybolt y Wyatt (1982): La reformulación es el "proceso de cambiar los datos numéricos para producir una forma [del problema] más manejable mentalmente. Este proceso deja la estructura del problema intacta” (p. 187). La traducción es el "proceso de cambiar la estructura matemática del problema por otra más manejable mentalmente" (p. 188). La compensación consiste en los "ajustes hechos para reflejar variaciones en los números debidas a la reformulación y a la traducción” (p. 189). En un segundo nivel de análisis, encontramos el redondeo, truncamiento y sustitución para los procesos de reformulación, cambio en el orden de las operaciones o de operación para los de traducción, y para los de compensación, cambios en datos o en resultado (Segovia, Castro, Rico y Castro, 1989; Levine, 1982). En subsiguientes niveles, pueden tenerse en cuenta otras variables, como la forma de operar la coma decimal (Gómez, 1995a), el grado de aproximación (redondeando a centenas, decenas, etc.), el trabajar con extractos en los redondeos o arrastrar los ceros (Reys y otros, 1982; Segovia y otros, 1989), aspectos específicos de las operaciones o los números intervinientes (Hanson y Hogan, 2000), etc. Este nivel de detalle aparece en Pañellas (2006), en que se llegan a diferenciar 103 tipos de estrategias de estimación.

Para profundizar en el conocimiento de la dificultad de los ítems de la prueba de estimación, vamos a abordar el análisis de los errores de los profesores al estimar. Previamente, dentro del ámbito de la estimación, conviene diferenciar tres términos relativos a los errores en matemáticas: precisión, razonabilidad y error. Segovia, Castro, Castro y Rico (1989) indican que el error "designa la diferencia o desviación que un valor aproximado tiene con respecto del valor exacto al que representa" (p. 85). Esta acepción de error como desviación es fundamental, pues la propia naturaleza de la estimación exige aceptar un cierto margen de error. Así, Reys y otros (1982) definen la tolerancia del error como una "comprensión del concepto de estimación que permite sentirse cómodo con cierto grado de error" (p. 198). El error relativo, o razón entre el error absoluto y el valor exacto, se utiliza para valorar la precisión de las estimaciones. En varios estudios (De Castro, 2002; Dowker, 1992; Hanson y Hogan, 2000; Levine, 1980, 1982) se han puntuado con un cero las estimaciones con porcentaje de error superior al 30\%. Este porcentaje aumenta al 40\% en algunas investigaciones en Educación Primaria (Liu, 2009). Siguiendo esta línea, consideramos imprecisas las estimaciones con error mayor del 30\%. La imprecisión no supone la presencia de un error en matemáticas, entendido como "conocimiento deficiente e incompleto" (Rico, 1995: 69). Por otra parte, Alajmi y Reys (2010) reconocen dos facetas fundamentales para la razonabilidad: a) las relaciones numéricas y el efecto de las operaciones y $b$ ) la practicidad, o sentido práctico en un contexto (por ejemplo, 6 entradas de cine no pueden costar 0,61 euros). Existe una gran diferencia entre razonabilidad y precisión. Una estimación puede tener un grado de precisión muy alto y no ser razonable (Alajmi y Reys, 2007 y 2010). Por ejemplo, no es razonable que 5 botellas de refresco de $1,95 €$ cuesten $10,01 €$, a pesar de que el porcentaje de error sea muy bajo. 


\section{Objetivos y pregunta de investigación}

Teniendo en cuenta los resultados citados en el apartado anterior, nos preguntamos por la dificultad relativa de las tareas de estimación en función del tipo de operación y de número. Conjeturamos, siguiendo los antecedentes del problema, que será clave la distinción entre decimales menores que 0,1 , decimales entre 0,1 y 1 , y decimales mayores que 1 y, dentro de la división, diferenciar si el dividendo es mayor o menor que el divisor. Así, nos planteamos los siguientes objetivos:

a) Estudiar la dificultad relativa de las tareas de estimación según el tipo de operación y de número. Dentro del tipo de operación, consideramos la multiplicación, la división A (dividendo mayor que divisor) y la división $\mathrm{B}$ (dividendo menor que divisor). En el tipo de número distinguimos los naturales, decimales mayores que 1 , decimales entre 0,1 y 1 , y los menores que 0,1 . Para abordar este objetivo, diseñaremos una prueba de estimación.

b) Analizar las estrategias de estimación de los estudiantes de Magisterio al estimar los resultados de cálculos multiplicativos con números decimales.

c) Elaborar un modelo para la clasificación de errores e imprecisiones (y términos afines al error) en estimación, considerando la estimación dentro de la resolución de problemas, y atendiendo especialmente a las peculiaridades de los procesos de estimación y de las operaciones con números decimales. Clasificar los errores de los estudiantes de Magisterio al estimar los resultados de multiplicaciones y divisiones con números decimales.

En el análisis de estrategias hemos seguido la terminología utilizada por Reys y otros (1982), considerando las estrategias de primeros dígitos, sustitución y fracciones. Hemos separado las estrategias de sustitución y las de fracciones, pues en este trabajo las estrategias con sustitución por números compatibles implican procesos de reformulación, mientras que las de fracciones suponen un proceso de traducción.

Por otra parte, para elaborar un modelo de clasificación de los errores en estimación, hemos atendido cuatro principios:

1. Considerar las tareas de estimación dentro del ámbito de la resolución de problemas.

2. Aplicar el marco teórico propuesto por Hiebert y Wearne (1986) para el estudio de las operaciones (y errores) con números decimales.

3. Tener en cuenta en el modelo la definición de estimación de Sowder (1988), y mostrar la relación del modelo con el modelo RTC de procesos de estimación de Reys y otros (1982).

4. Ajustar el modelo empleando resultados de investigaciones anteriores sobre errores en estimación con multiplicaciones y divisiones (De Castro, 2002; Levine, 1980).

El modelo desarrollado para los errores adopta una forma de modelo de fases, resultado de cribar la información de la revisión de modelos de fases en resolución de problemas (Castro, 1991: 39), eligiendo las más acordes a la definición de estimación en cálculo de Sowder (1988) y atendiendo también al modelo de Hiebert y Wearne (1986). La opción finalmente adoptada consiste en considerar errores en la fase de interpretación, en la que se atribuyen significados a los símbolos que aparecen en las operaciones, y se producen procesos de reformulación y traducción a través de destrezas de aproximación (como el redondeo, truncamiento, sustitución por fracciones, etc.) y compensaciones previas; la fase de ejecución, en la que se produce el cálculo mental, en el que un ingrediente fundamental es la determinación del orden de magnitud del resultado mediante la aplicación de reglas para ubicar la coma decimal, y la fase de evaluación, en la que se valora la razonabilidad del resultado. 


\section{MÉTODO}

Hemos empleado en el estudio una metodología mixta, con dos partes complementarias: la primera, de tipo cuantitativo, tiene un diseño factorial de medidas repetidas, en la que hemos diseñado, administrado y analizado estadísticamente una prueba de estimación; la segunda, de tipo cualitativo, está dedicada al análisis de estrategias y errores, a partir de una entrevista semiestructurada.

\section{Participantes}

Han participado 131 estudiantes de Magisterio pertenecientes a cinco grupos (Grupo 1 y Grupo 3, alumnos de primer curso de Maestro de Educación Primaria; Grupo 2, alumnos tercer curso de Maestro de Educación Primaria; y Grupo 4 y Grupo 5, alumnos de primero de Educación Infantil). De ellos, 108 pertenecían al CSEu La Salle, centro privado adscrito a la Universidad Autónoma de Madrid, y los 23 restantes (Grupo 3) eran estudiantes de la Facultad de Ciencias de la Educación de la Universidad de Granada. Los participantes no han sido elegidos aleatoriamente de ninguna población, sino que constituyen una muestra por conveniencia. Los alumnos de los grupos 1 y 2 recibieron enseńanza sobre estimación durante un periodo de 10 horas.

\section{La prueba de estimación}

Hemos diseñado una prueba de estimación, cuyos ítems responden a un diseño factorial de dos factores: las variables "tipo de número" con cuatro niveles y "tipo de operación” con tres niveles. Los tipos de números que corresponden a los cuatro niveles son: N1-números naturales; N2-números decimales mayores que uno; N3-números decimales entre 0,1 y 1, y N4-números decimales menores que 0,1. Los tres tipos de operaciones empleadas son: multiplicación, división tipo A (con el dividendo mayor que el divisor) y división tipo B (dividendo menor que el divisor). Al cruzar estas dos variables, obtenemos doce tipos de ítems, de cada uno de los cuales hemos incluido dos versiones para observar la fiabilidad de los resultados. En la tabla 1 figuran los ítems que componen la prueba de estimación, clasificados según el tipo de operación y de número. La prueba de estimación fue diseñada con dos formas paralelas del mismo test. Cada ítem impar de la prueba se propone como equivalente al ítem siguiente. El coeficiente de fiabilidad obtenido tiene un valor de $\mathrm{r}=0,79$.

Tabla 1.

Ítems de la prueba de estimación según el tipo de operación y tipo de número

\begin{tabular}{|c|c|r|r|}
\hline & \multicolumn{3}{|c|}{ Tipo de operación } \\
\hline Número & \multicolumn{1}{|c|}{ Multiplicación } & \multicolumn{1}{|c|}{ División A } & \multicolumn{1}{c|}{ División $B$} \\
\hline \multirow{2}{*}{$\mathrm{N}_{1}$} & $(1) 46 \times 771$ & (3) $968 \div 24$ & $(5) 86 \div 222$ \\
& $(2) 58 \times 244$ & $(4) 354 \div 88$ & $(6) 36 \div 258$ \\
\hline \multirow{2}{*}{$\mathrm{N}_{2}$} & $(7) 78,4 \times 89,5$ & (9) $85,9 \div 3,42$ & $(11) 9,88 \div 25,6$ \\
& $(8) 34,1 \times 47,2$ & $(10) 96,2 \div 6,25$ & $(12) 8,85 \div 42,6$ \\
\hline \multirow{2}{*}{$\mathrm{N}_{3}$} & $(13) 2,57 \times 0,72$ & $(15) 0,962 \div 0,25$ & (17) $0,37 \div 0,543$ \\
& $(14) 0,45 \times 7,85$ & $(16) 0,747 \div 0,35$ & (18) $0,63 \div 0,785$ \\
\hline \multirow{2}{*}{$\mathrm{N}_{4}$} & $(19) 0,025 \times 776$ & (21) $0,46 \div 0,066$ & (23) $0,059 \div 0,23$ \\
& $(20) 852 \times 0,048$ & (22) $0,68 \div 0,024$ & (24) $0,086 \div 0,42$ \\
\hline
\end{tabular}


La prueba se administró mediante ordenador en un aula de informática, donde los participantes entraban sin llevar nada consigo (para evitar procedimientos no mentales de cálculo), durante una sesión de dos horas de duración. Los 24 ítems de la prueba aparecían en orden aleatorio en la pantalla del ordenador. Los alumnos no tenían limitación de tiempo para responder a cada ítem, pero se indicó que debían dar su respuesta con rapidez. La duración media de la prueba fue de 24 minutos y 27 segundos.

\section{Las entrevistas}

Los participantes entrevistados fueron 26 de los 33 alumnos del Grupo 1. Fueron elegidos por su disponibilidad, pues estudiaban el curso Didáctica de las Matemáticas para la Educación Primaria, con la estimación en cálculo entre sus contenidos, y la prueba de estimación y la entrevista se integraban dentro de las actividades del curso. Los alumnos fueron entrevistados días más tarde de finalizar el periodo de instrucción sobre estimación y de realizar la prueba de estimación, administrada inmediatamente después de este.

Los 26 alumnos entrevistados fueron divididos al azar en dos grupos. En el primero de ellos, se pidió dar una estimación para los ítems impares de la prueba de estimación, explicando el procedimiento que habían seguido para producir el resultado. En el segundo, se utilizaron los ítems pares. Esta opción se tomó aprovechando que las dos partes de la prueba se habían diseñado para ser equivalentes y que una entrevista con todos los ítems de la prueba resultaría excesivamente larga. Las entrevistas se realizaron individualmente, en una habitación aislada, con el entrevistador sentado junto al alumno, y ambos delante de un ordenador, con el mismo software empleado en la prueba. Las entrevistas fueron registradas con una grabadora y transcritas para su posterior análisis.

\section{RESULTADOS}

Presentamos por separado los resultados obtenidos para cada uno de los objetivos planteados en el estudio. En primer lugar, exponemos los resultados del análisis de las puntuaciones de los ítems de estimación incluidos en la prueba. En segundo lugar, desarrollamos el análisis de estrategias. Para finalizar, exponemos el análisis de errores.

\section{Análisis de la puntuación de la prueba}

La prueba de estimación, compuesta por 24 ítems (tabla 1), ha sido administrada a 131 estudiantes de Magisterio, lo que supone un total de 3.144 estimaciones. De ellas, 1.730 (un 55\%) han sido clasificadas como imprecisas (error mayor del 30\%). Por el contrario, 1.414 respuestas (un 45\%) han tenido un grado de precisión aceptable (error menor o igual del 30\%).

Tabla 2.

Puntuaciones medias de la prueba de estimación según el tipo de operación y tipo de número

\begin{tabular}{|c|c|c|c|c|c|c|}
\hline & \multicolumn{2}{|c|}{ Multiplicación } & \multicolumn{2}{c|}{ División $A$} & \multicolumn{2}{c|}{ División $B$} \\
\hline Número & $M$ & $D E$ & $M$ & $D E$ & $M$ & $D E$ \\
\hline N1 & 1,48 & 0,09 & 1,41 & 0,09 & 1,30 & 0,07 \\
\hline N2 & 1,50 & 0,11 & 1,65 & 0,09 & 1,15 & 0,09 \\
\hline N3 & 0,77 & 0,08 & 0,90 & 0,10 & 0,73 & 0,08 \\
\hline N4 & 0,56 & 0,08 & 0,66 & 0,09 & 0,78 & 0,08 \\
\hline
\end{tabular}


En la prueba de estimación se ha empleado un diseño factorial de tres factores con medidas repetidas en dos de ellos. Los dos factores intrasujetos son el tipo de operación y el tipo de número. El factor intersujetos es el grupo. En este diseño, el factor operación tiene tres niveles: multiplicación, división de un número por otro menor (división $\mathrm{A}$ ) y división de un número por otro mayor (división $\mathrm{B}$ ); el factor número tiene por niveles los números enteros, decimales mayores que 1 , decimales entre 0,1 y 1 , y decimales menores que 0,1 . La variable dependiente utilizada es la puntuación (3 puntos si el porcentaje de error es menor del 10\%; 2 puntos si el porcentaje varía entre el 10 y el 20\%; 1 punto si está entre el 20 y el $30 \%$, y 0 puntos en cualquier otro caso). En este trabajo, el término dificultad no lo utilizamos en el sentido habitual de "índice de dificultad" propio de la psicometría. Consideramos un conjunto de ítems más difícil que otro cuando su puntuación media sea significativamente inferior. La dificultad relativa de los ítems se establece mediante las comparaciones por pares del análisis de varianza.

Mediante un análisis de la varianza hemos obtenido que tanto el factor tipo de operación $(\mathrm{F}=3,67$; gl. $=1,91 ; \mathrm{p}<0,05)$ como el factor tipo de número ( $\mathrm{F}=80,67$; gl. 2,60; $\mathrm{p}<0,001)$ tienen un efecto significativo sobre la variable dependiente puntuación. No se ha encontrado efecto de interacción del tipo de número y operación sobre la puntuación, independiente del grupo.

\section{Influencia del tipo de número sobre la puntuación}

Dado que el factor tipo de número ha tenido un efecto significativo sobre la puntuación, recurrimos a las comparaciones múltiples con el ajuste de Bonferroni, empleando la D para representar la diferencia entre las medias para los niveles de la variable tipo de número. Los ítems con decimales menores que 0,1 han tenido una puntuación media inferior a los ítems con decimales entre 0,1 y 1 ( $\mathrm{D}=0,13, \mathrm{p}<$ $0,05)$, menor a la de los ítems con decimales mayores que $1(\mathrm{D}=0,76, \mathrm{p}<0,001)$ y menor que los ítems con números naturales $(\mathrm{D}=0,73, \mathrm{p}<0,001)$. Los ítems con números decimales menores que 1 han tenido una puntuación media menor que aquellos con decimales mayores que 1 ( $\mathrm{D}=0,63, \mathrm{p}<$ $0,001)$ y que los ítems con números naturales $(\mathrm{D}=0,59, \mathrm{p}<0,001)$. En la figura 1 observamos que las diferencias de dificultad, citadas en el párrafo anterior, se han producido para todos los grupos de participantes del estudio.

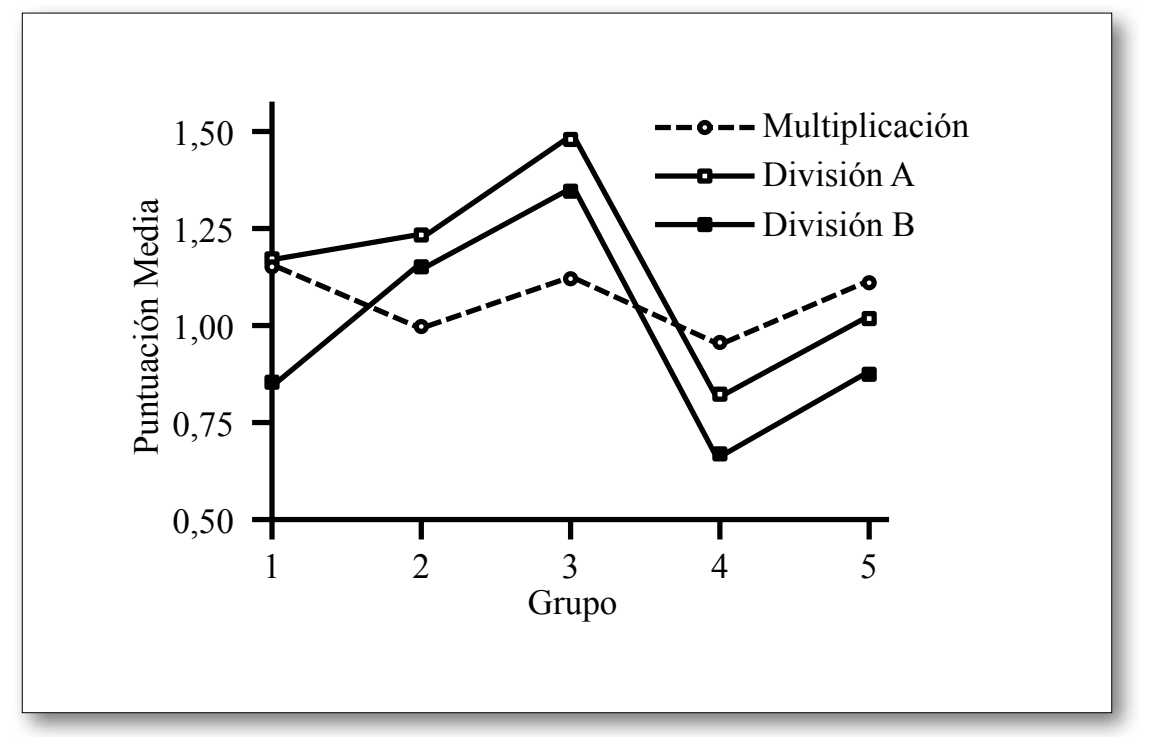

Fig. 1. Puntuaciones medias de los ítems según el tipo de número y el grupo 


\section{Influencia del tipo de operación sobre la puntuación}

Puesto que el tipo de operación ha tenido un efecto significativo sobre la puntuación, hemos realizado las comparaciones por pares entre las medias de las puntuaciones, para los distintos niveles del factor, con el ajuste de Bonferroni. Los ítems de estimación con divisiones de tipo B, con el dividendo menor que el divisor, han tenido una puntuación media $(\mathrm{M}=0,99 ; \mathrm{DE}=0,06)$ significativamente inferior $(\mathrm{D}=0,16 ; \mathrm{p}<0,01)$ a las divisiones de tipo $\mathrm{A}(\mathrm{M}=1,15 ; \mathrm{DE}=0,06)$. En la figura 2 vemos que esta diferencia de dificultad entre los dos tipos de divisiones se ha producido en los 5 grupos.

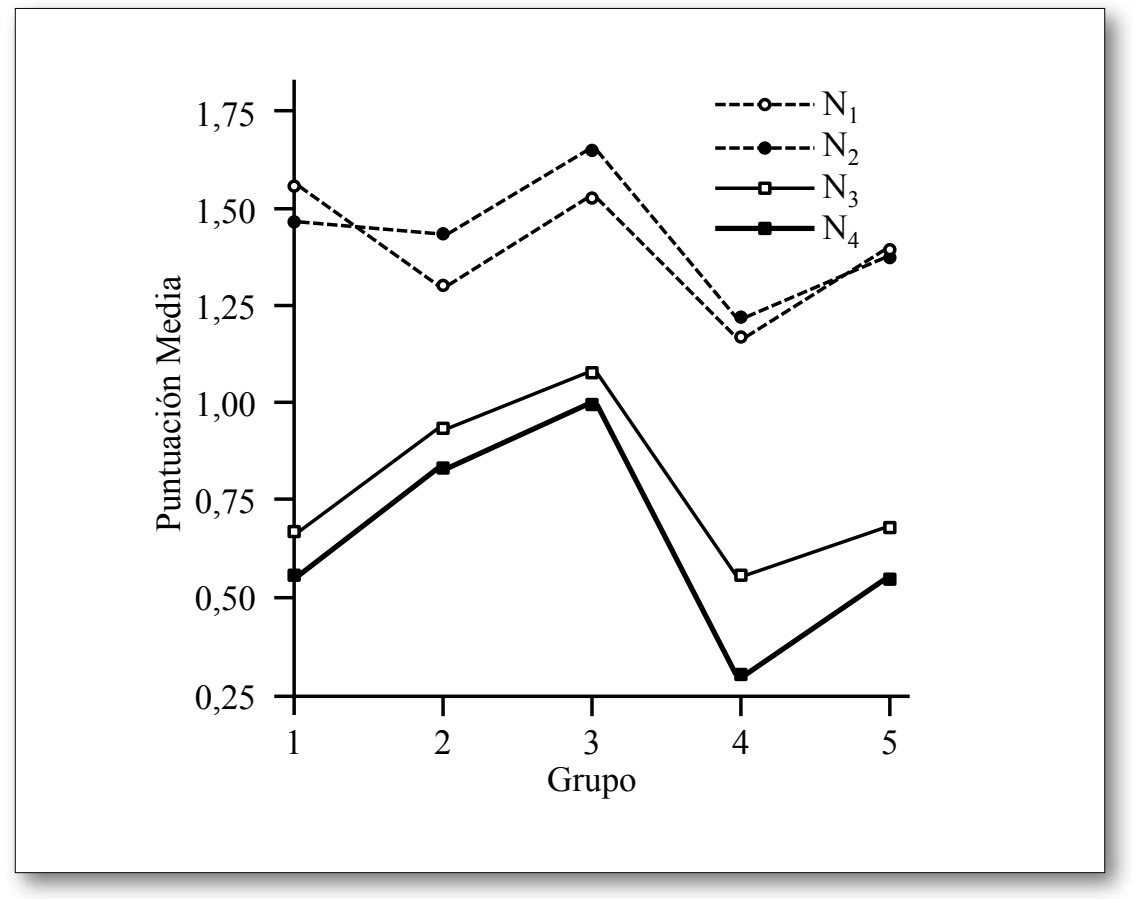

Fig. 2. Puntuaciones medias de los ítems según el tipo de operación y el grupo

\section{Análisis de estrategias de estimación}

$\mathrm{Al}$ analizar las estrategias de estimación, hemos encontrado cuatro estrategias básicas: primeros dígitos, sustitución, fracciones y algoritmo simplificado. Cada estrategia aparece ilustrada con un fragmento de transcripción de entrevista. Hemos puesto diversas variantes de cada estrategia para mostrar que las estrategias pueden analizarse en distintos niveles.

\section{Primeros dígitos}

La característica principal de esta estrategia consiste en la reducción de la estimación al uso de hechos numéricos básicos, implicando solo a los dígitos de cada número de mayor valor posicional y a la determinación del orden de magnitud del resultado. Normalmente, se produce una reformulación mediante una destreza de aproximación, redondeo o truncamiento, pero en el caso de la división a veces se lleva a cabo sin reformulación. Presentamos ejemplos de las variantes que admite esta estrategia según se aplique una destreza de aproximación u otra, e incluso sin proceso de reformulación previo al cálculo. También mostramos las diferentes formas de operar la coma decimal. 
Tarea: $\quad 34,1 \times 47,2$

Alumno 2: 34,1 lo redondeo a 30, y 47,2 a 50. Tres por cinco, 15. 1500 .

Aquí se emplea el redondeo (47,2 a 50 y 34,1 a 30), transformando los decimales en naturales y evitando el uso de la coma decimal en el cálculo.

En la mista tarea, en el caso siguiente, el redondeo también se puede utilizar posteriormente a la multiplicación de ambos datos por potencias de 10 (472 a 500 y 341 a 300) para operar sin la coma decimal que luego aplica en el resultado:

Tarea: $\quad 34,1 \times 47,2$

Alumno 28: Multiplico por 10. Serían 341 por 472.341 lo voy a redondear a 300 y 472 a 500.5 por 3, 15 y cuatro ceros, y le quito dos por las comas, 1.500 .

En el caso siguiente se emplea el truncamiento de ambos datos como destreza de aproximación:

Tarea: $\quad 46 \times 771$

Alumno 9: Esto tiende a 50. Esto a 1.000. Entonces sería... Así, o bien también por 4 por 7, 28, con tres ceros, sería... 28.000.

En el caso siguiente, se cambia 89,5 por 100 (sustitución por potencias de diez) en lugar de redondear a las decenas (90) o truncar el dato inicial (80). La proximidad se sacrifica en beneficio de la sencillez de cálculo.

Tarea: $\quad 78,4 \times 89,5$

Alumno 12: Redondeo esto a 80 y esto a 100. Entonces 80 por 100 son 8000.

Finalmente, a veces no se utiliza ninguna destreza de aproximación y no hay sustitución de los datos iniciales sino en los que surgen de la transformación de los decimales en naturales. Se determina la primera cifra del cociente observando los primeros dígitos (37 y 5).

Tarea: $\quad 0,37 \div 0,543$

Alumno 20: Corro la coma tres a la derecha en el divisor; tres a la derecha en el dividendo. 370 entre 543, 0,7 .

\section{Sustitución}

También se producen, en los procesos de reformulación, sustituciones por "números compatibles", habituales en situaciones de división, en que se sustituyen dividendo y divisor por dos números con relación de múltiplo y divisor:

Tarea: $\quad 968 \div 24$

Alumno 3: Aquí he puesto 1.000 y lo he hecho entre 25 . Y como hay 4 cada 100, 4 por 10, 40 .

\section{Fracciones}

Aunque esta estrategia también es de sustitución, dadas las características específicas de esta, el empleo de fracciones y la relevancia de su uso, la consideramos con esta denominación. El elemento distintivo es la sustitución de un número decimal por una fracción, lo que afecta a la fase de cálculo mental y al modo de operar la coma decimal. En los siguientes ejemplos vemos tres formas diferentes de operar la coma decimal en el dato no sustituido por una fracción, dando cuenta de tres variantes de la estrategia. 
Tarea: $\quad 2,57 \times 0,72$

Alumno 20: Esto multiplico. Voy a utilizar este número como 250. Este número como 0,75. 250 por 0,75 es como multiplicar 250 por 3/4. 250 entre 4. Bueno, voy a hacer primero por 3. Son 750 . Entre 4, son un poco menos de 140 . Y tengo que quitar dos comas. O sea, 1,4.

El cálculo inicial $2,57 \times 0,72$ se sustituye por $(3 / 4$ de 250$) \div 100$. Para operar la coma decimal en 2,57 se multiplica por 100 y se deja pendiente la división por 100 para el final. En el ejemplo siguiente, al redondear implícitamente 7,85 a 8, se elimina la coma decimal al aplicar la destreza de aproximación.

Tarea: $\quad 0,45 \times 7,85$

Alumno 2: $\quad 0,45$ lo redondeo a 0,5 que es $1 \frac{1}{2}$. Y lo multiplico por... no sé. A ver... Bueno, sí. Un medio y por eso $[7,85]$ pues... 4 más o menos.

A continuación, se utiliza una tercera alternativa para operar la coma decimal. La coma decimal no se elimina, multiplicando el número por una potencia de diez, ni aplicando una destreza de aproximación, sino que se arrastra, operando por separado la parte entera y la parte decimal del número. Así, al hacer la mitad de 7,85 , parece hacerse por un lado la mitad de la parte entera $(1 / 2 \mathrm{de} 7 \approx 3) \mathrm{y}$, por otro, la mitad de la parte decimal $(1 / 2$ de $85 \approx 43)$.

Tarea: $\quad 0,45 \times 7,85$

Alumno 6: $\quad 0,45$ pues... lo paso... Esto es 0,5 , que sería la mitad al multiplicarlo por esto $[7,85]$ y sería 3,43 , creo.

En un nivel más detallado del análisis, en la estrategia de fracciones, podemos centrarnos en las destrezas de aproximación (y el grado de aproximación) aplicadas al dato no sustituido por una fracción. Así, tenemos un truncamiento a las decenas (257 a 250), un "redondeo no estándar" $(0,72$ a 0,75) previo a la sustitución del 0,75 por $3 / 4$ o un redondeo de 7,85 a 8 .

\section{Algoritmo alternativo}

Esta estrategia se ha producido en divisiones y parece una adaptación de una estrategia de cálculo mental a situaciones de estimación. Consiste en descomponer el dividendo como producto de dos factores, buscando que uno de ellos sea un múltiplo aproximado del divisor. A continuación se calcula cuántas veces cabe el divisor en ese factor (bien a través de sumas reiteradas del divisor o de productos del divisor por otro número), y luego se multiplica el resultado por el otro factor. Las relaciones de múltiplo y divisor se emplean de forma aproximada.

En el ejemplo siguiente, la operación de la coma decimal transforma la operación inicial en $680 \div$ 24. Después se cambia por $700 \div 25=(7 \times 100) \div 25$, y esto, a su vez, por $7 \times(100 \div 25)=7 \times 4=$ 28. Dado que apenas se alteran los datos iniciales, y que su cambio incluye además una compensación previa involuntaria, el porcentaje de error es solo del 1,2\%.

Tarea: $\quad 0,68 \div 0,024$

Alumno 27: 680 entre 24. 24 [inaudible] 25. 680 entre 24. 24 por 4, 100, por 7, 28. Yo creo que son 28.

Otro ejemplo de esta estrategia incluye un pequeño cambio con respecto al anterior. El alumno 14 realiza la división de $100 \div 25$ mediante una suma para llegar a la conclusión de que "100 tiene 4 veinticincos". La estrategia puede sintetizarse del modo siguiente: 9,88 $\div 25,6$ da lugar a un resultado que comienza por " 0 ,". A continuación, se debe resolver $98,8 \div 25,6 \approx 100 \div 25$. Aquí es donde el alumno resuelve la división a través de una suma. 
Tarea: $\quad 9,88 \div 25,6$

Alumno14: Me parece que si pongo un cero de resultado, eso quiere decir que bajo la coma. O sea, que en lugar de 9,88, puedo poner 98,8. Y a mí me da que eso lo hago si pongo un cero coma en el resultado. Entonces yo sigo así. Y 98,8 entre 25,6... Vamos a redondear. Claro. Y 98,8 ya directamente ¿a qué pasa? A 100 . Y 100 entre 25.100 entre 25.2 por $5 \ldots 5$ por $4 \ldots$ A ver. 100 entre 25.25 más 25, 50, 75 y 100. 4 . 100 tiene 4 veinticincos. Porque 4 por 5, 20; 4 por 2,8 y 2,10 . Vale.

Una variante de esta estrategia consiste en determinar el resultado mediante tanteos sucesivos, utilizando la descomposición aditiva del dividendo $(96=90+6)$ y recuperando el resultado $6 \times 15=90$. En este caso (como ya ocurre en el ejemplo anterior) no hay descomposición factorial del dividendo, aunque sí aditiva, por eso se considera una "variante" de la estrategia.

Tarea: $\quad 96,2 \div 6,25$

Alumno27: 6 por 15 serían 90, más otro 6, 96. Sería 16 .

\section{Análisis de los errores}

En la figura 3 proponemos el modelo elaborado para el análisis de errores (a la izquierda), cuya procedencia hemos explicado en el apartado de objetivos y su encaje con las categorías de errores encontradas (a la derecha). Utilizando este modelo de clasificación hemos estudiado la fiabilidad del proceso de codificación valorando el grado de acuerdo interjueces. Así, sobre una muestra aleatoria del 30\% de las transcripciones de las entrevistas, se ha obtenido un valor 0,81 para el coeficiente Kappa de Cohen, lo que indica una concordancia "casi perfecta" entre codificadores. A continuación, presentamos la definición de todas las categorías de errores, acompañadas de ejemplos procedentes de las entrevistas.

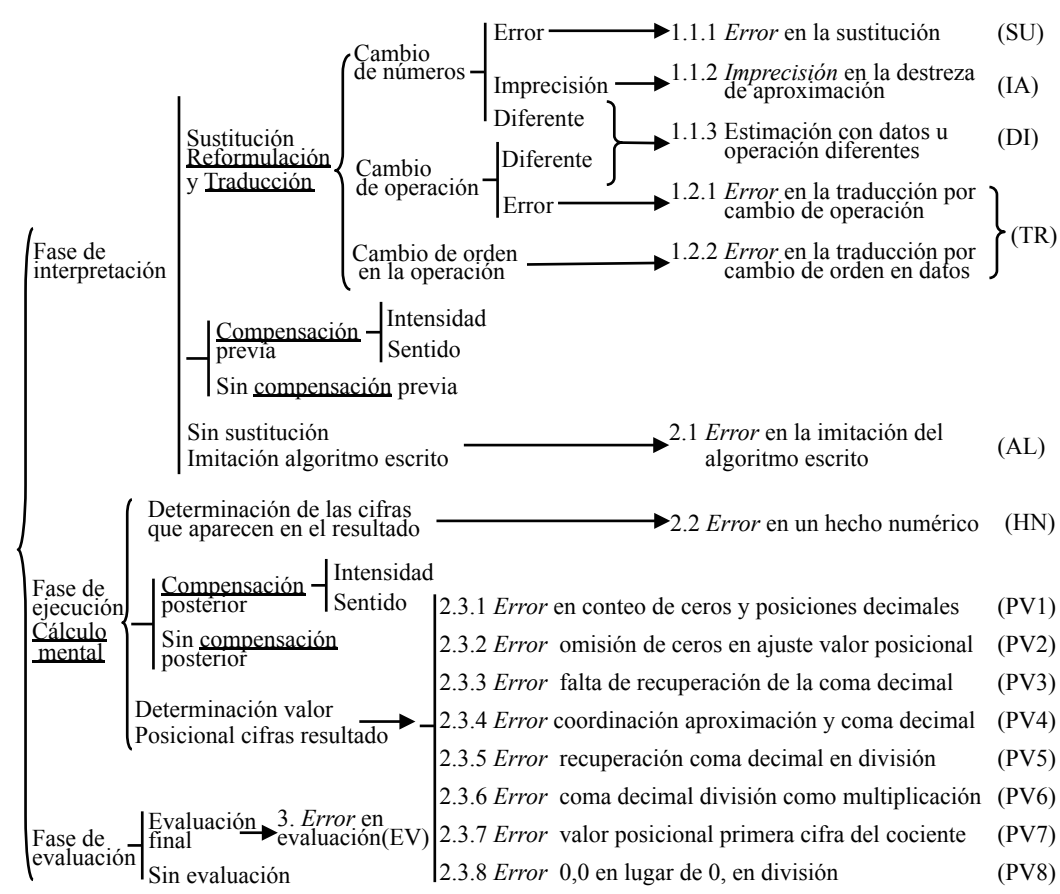

Fig. 3. Modelo para el análisis de errores 


\section{Errores en la fase de interpretación}

Error en la sustitución (su). Consiste en aplicar incorrectamente una destreza de aproximación (redondeo, truncamiento, sustitución por potencias de diez) o una sustitución de un decimal por una fracción. Por ejemplo, sustitución de 0,06 por 0,5 . En el segundo caso, se puede dar una adaptación incorrecta de las sustituciones por fracciones $(0,5$ por $1 / 2$ y 0,25 por $1 / 4)$ a 0,05 o a 0,025 , como en la sustitución de 0,025 por $1 / 4$.

Imprecisión en la aproximación (IA). Aplicar correctamente una destreza de aproximación, dando lugar a una estimación imprecisa (error mayor 30\%). Por ejemplo, sustituir $2,57 \times 0,72$ por $3 \times 1$ (error del $62,1 \%$ ), o $96,2 \div 6,25$ por $100 \div 10$ (error del $35,0 \%$ ), o $0,059 \div 0,23$ por $0,1 \div 0,2$ (error del $94,9 \%)$.

Estimación con datos u operación diferentes a los propuestos (DI). Realizar una estimación con alguno de los datos o con la operación diferente de los propuestos. Por ejemplo, para estimar el resultado de $0,025 \times 776$, el alumno divide, en lugar de multiplicar, y da una estimación de 0,00003

Error en la traducción (TR). En una sustitución de un decimal por una fracción, cambio de la operación propuesta por la aplicación de la fracción, como operador, al otro dato. Es decir, sustitución de $a / b \div n$ o $n \div a / b$ por $(a \times n) \div b$. También se considera error de traducción, por cambio en el orden de los datos, que se ha de cambiar en una división, el papel de dividendo y divisor. Ejemplo de estos casos en la investigación han sido el cambio de $0,37 \div 0,543$ por $0,4 \div 0,5$, y esto por $1 / 2$ de $0,4=0,2$. También cambiar $0,63 \div 0,785$ por $0,66 \div 0,780$, y esto por $(2 / 3$ de 780$) \div 1000=0,52$. Ejemplo de error de traducción por cambio en el orden de los datos es hacer $86 \div 222 \approx 100 \div 222=2,22$.

\section{Errores en la fase de ejecución}

Error en la imitación del algoritmo escrito (AL). Se produce en la ejecución de la imitación mental del algoritmo escrito. En la estimación de $0,37 \div 0,543$, el alumno 26 opera según se indica en la figura 2 . Como vemos en ella, y en la transcripción, la primera cifra del cociente se multiplica solo por la cifra de las decenas del divisor, y la segunda cifra del cociente, por la cifra de las unidades.

Entrevistador: $\quad 0,37 \div 0,543$

Alumno 26: Quitaríamos la coma y queda 37 entre 54,3. Entonces, como es menor, pues pondría aquí un 0 y abajo un $0, \ldots$ y entonces... pues ya podría dividir y entonces... sería 37 entre 5 . No. A ver... 37 entre 54, y entonces cogemos 37 entre 5 a 7 por 5, 35 y llevo 2. Serían 20 entre 4 , serían $5 \ldots$

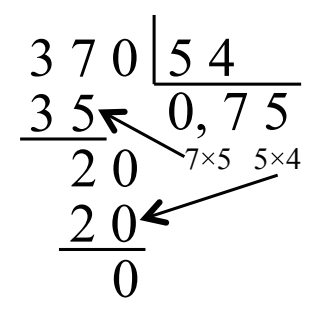

Fig. 4. Imitación mental del algoritmo escrito. 
Error en un hecho numérico (HN). Error en la recuperación de un hecho numérico, dé lugar o no a una estimación precisa. Por ejemplo, $2,57 \times 0,72 \approx 3 \times 1=1$ ( $46,0 \%$ de error) o $46 \times 771 \approx 50 \times 800$ $=56.000$ (haciendo $5 \times 8=56$, con un $57,9 \%$ de error).

Error en el conteo de ceros y posiciones decimales (VP1). Utilizar una destreza de aproximación y una técnica correcta para determinar el valor posicional, equivocándose en el conteo de los ceros o las posiciones decimales del resultado. 96,2 $\div 6,25 \approx 10.000 \div 600 \approx 10.000 \div 500=200$ (200 en vez de 20$)$. $852 \times 0,048 \approx 900 \times 40 / 1.000=(900 \times 40) \div 1.000=3.600 \div 1.000=3,6(3.600$ en vez de 36.000).

Error de omisión de ceros en el ajuste del valor posicional (VP2). Utilizar una técnica de aproximación y omitir, en la determinación del valor posicional del resultado, los ceros provenientes de la destreza de aproximación. Por ejemplo, $2,57 \times 0,72 \approx(300 \times 70) \div(100 \times 100)=3 \times 7 \times 1.000 \div 10.000=$ 0,0021 . Se omiten los 3 ceros provenientes de $300 \times 70$.

Error de falta de recuperación de la coma decimal (VP3). Multiplicar los datos por potencias de diez para eliminar la coma decimal, utilizar una técnica de aproximación y omitir, en la determinación del valor posicional del resultado, la división por la unidad seguida de tantos ceros como posiciones decimales había en los datos de partida. 78,4 × 89,5 $\approx(700 \times 800) \div 100=(7 \times 8) \times 10.000 \div 100=$ 560.000. Se omite la división final por 100.

Error en coordinación de la aproximación con la coma decimal (VP4). Aplicar una destreza de aproximación eliminando cifras decimales. Después, en el ajuste del valor posicional, se tienen en cuenta las cifras decimales originales. Por ejemplo, $34,1 \times 47,2 \approx 30 \times 50=1.500$. Después, divide por 100, por las 2 cifras decimales iniciales, sin advertir que en el redondeo han sido eliminadas. La estimación es 15 .

Error de recuperación impropia de la multiplicación o división por una potencia de diez (VP5). Multiplicar o dividir dividendo y divisor por una potencia de 10 para eliminar la coma decimal o simplificar el cálculo, calcular el cociente y dividirlo o multiplicarlo después por la misma potencia de 10 utilizada al principio (cuando esto no es necesario por haber obtenido una división equivalente). Por ejemplo, $0,46 \div 0,066 \approx 50 \div 7=7$. Ahora se divide por 100 (dado que al principio se multiplicó por 100), para una estimación de 0,07 .

Error de operar la coma decimal en la división como en la multiplicación (VP6). Se dividen los números ignorando las comas decimales y después se divide el resultado por la unidad seguida de tantos ceros como posiciones decimales hay entre los dos números (como al multiplicar). $0,46 \div 0,066 \approx 46$ $\div 6 \approx 7$. Después se divide por 10.000 porque hay 5 posiciones decimales entre los dos datos, para una estimación de 0,00007 .

Error en la determinación del valor posicional de la primera cifra del cociente (VP7). En divisiones en las que no se utiliza destreza de aproximación, se determina la primera cifra distinta de cero del cociente, pero se falla en establecer su valor posicional. $0,68 \div 0,024=680 \div 24 \approx 2$ (en lugar de 20 , que sería lo correcto).

Error de 0,0 en lugar de 0, en la división (VP8). Empezar la división de un número por otro mayor poniendo " 0,0 " cuando corresponde solo " 0 ,". El segundo 0 parece hacer el efecto de bajar la siguiente cifra del dividendo. $86 \div 222 \approx 100 \div 200=0,05$ (en lugar de 0,5 , que sería lo indicado).

\section{Errores en la fase de evaluación}

Errores en la evaluación (Ev). El error de evaluación consiste en la sustitución de un proceso de estimación correcto, que concluye con una estimación razonable, tras una evaluación incorrecta de dicho proceso (error Ev), por un proceso de estimación incorrecto, en que se produce un error diferente al de evaluación. Por ejemplo, hacer $86 \div 222 \approx 90 \div 200=9 \div 20 \approx 0,4$ para rectificar después poniendo $9 \div 20 \approx 0,04$ (error PV8). 


\section{Otros errores}

Otros errores e imprecisiones (от). Son errores que no entran en las categorías anteriores y pueden darse en cualquier fase. Hacer $86 \div 222 \approx 100 \div 222=2,22=2, \hat{2}$. En la primera igualdad hay un error de traducción, al cambiar los papeles de dividendo y divisor. En la segunda, un error OT, al igualar un número con dos cifras decimales con el decimal periódico propuesto a continuación. Otro ejemplo de error от es hacer $0,025 \times 776 \approx 776$, porque " 0,025 no se puede sustituir por 1 , pero multiplicar por un número tan pequeño no puede variar mucho el resultado" (alumno 14), lo que parece una generalización incorrecta de la propiedad de multiplicar por 1 números menores que 1 , considerados como "pequeños".

En la tabla 3 vemos un resumen de los errores encontrados en este trabajo, clasificados por la fase en la que se produce el error, el tipo de error, tipo de operación y de número. Para facilitar la lectura, se han eliminado errores de categorías con uno o dos casos, y los errores en evaluación y en la categoría от, que no están asociados a ningún tipo de operación o de número. Destacamos que 89 de los 130 errores $(68,5 \%)$ se han producido con números menores que 1 o menores que 0,$1 ; 79$ de los errores $(60,8 \%)$ se han dado en el ajuste de la coma decimal (categorías VP1 a VP8), y que el tipo VP8, específico de la división $B$, que se produce al comenzar una división por “ 0,0 ” en vez de " 0 ,", ha sido el error más frecuente (21 errores, un $16,2 \%$ del total).

Tabla 3.

Errores en la estimación según el tipo de operación y tipo de número

\begin{tabular}{|c|c|c|c|c|c|c|c|c|c|c|c|c|c|c|}
\hline \multirow[b]{2}{*}{ Fase } & \multirow[b]{2}{*}{ Errores } & \multicolumn{4}{|c|}{ Multiplicación } & \multicolumn{4}{|c|}{ División A } & \multicolumn{4}{|c|}{ División $B$} & \multirow[b]{2}{*}{ Total } \\
\hline & & $N_{1}$ & $N_{2}$ & $N_{3}$ & $N_{4}$ & $N_{1}$ & $N_{2}$ & $N_{3}$ & $N_{4}$ & $N_{1}$ & $\mathrm{~N}_{2}$ & $N_{3}$ & $N_{4}$ & \\
\hline \multirow{4}{*}{ Interpretación } & $S U$ & & & & 5 & 1 & & 2 & 1 & & 1 & & 1 & 11 \\
\hline & $I A$ & & & 3 & 1 & & 1 & & & & & 1 & 1 & 7 \\
\hline & $D I$ & & & 2 & 2 & & 1 & 1 & & & 2 & 2 & & 10 \\
\hline & $T R$ & & & & & 2 & & 5 & 1 & 1 & 2 & 2 & & 13 \\
\hline \multirow{11}{*}{ Ejecución } & $A L$ & & & & & & 1 & 1 & & 1 & & & & 3 \\
\hline & $H N$ & 1 & & 2 & & & & & 1 & 1 & & 2 & & 7 \\
\hline & $V P 1$ & 3 & 1 & 3 & 3 & 1 & 1 & 2 & & & 1 & & & 15 \\
\hline & $V P 2$ & & & 2 & 4 & & & & & & & & & 6 \\
\hline & $V P 3$ & & 1 & & 1 & & & & & & & & & 2 \\
\hline & VP4 & & 3 & 2 & 4 & & & & & & & & & 9 \\
\hline & VP5 & & & & & 2 & & 4 & 4 & & & & & 10 \\
\hline & VPG & & & & 1 & & & 2 & 1 & & & & 3 & 7 \\
\hline & $V P 7$ & & & & & & 4 & 1 & 4 & & & & & 9 \\
\hline & VP8 & & & & & & & & & 4 & 5 & 6 & 6 & 21 \\
\hline & Total & 4 & 5 & 14 & 21 & 6 & 8 & 18 & 12 & 7 & 11 & 13 & 11 & 130 \\
\hline
\end{tabular}

\section{CONCLUSIONES E IMPLICACIONES}

De nuestro análisis se puede concluir que las estimaciones planteadas no son especialmente difíciles para los estudiantes de Magisterio, como atestigua el 45\% de estimaciones aceptables dadas por los participantes, cuando un $60,3 \%$ de ellos no ha recibido instrucción sobre estimación. Sin embargo, sí que plantean dificultades los números decimales (en especial los menores que 1), las divisiones de un 
número por otro mayor, y el ajuste del valor posicional al operar con decimales. En particular, hemos visto que 8 de las 17 categorías de error encontradas en este trabajo corresponden a errores en el ajuste del valor posicional (muchos de ellos, al operar la coma decimal), algunos de los cuales habían sido ya descritos en trabajos de cálculo mental con decimales (Gómez, 1995b), y otra categoría (los errores de traducción, TR) se debe a las dificultades de operar naturales con fracciones y a la de dividir un número por otro mayor.

Ha resultado importante la distinción entre las divisiones A y B. Las divisiones con el dividendo menor que el divisor, además de ser significativamente más difíciles, producen errores específicos al estimar, como el de comenzar la estimación con “0,0" en lugar de “0," (error VP8). Estos resultados son coherentes con los que hemos reseñado en la introducción, provenientes de la investigación en problemas de estructura multiplicativa (Castro y Castro, 1996; De Corte y Verschaffel, 1996). La distinción, dentro de los decimales menores que 1 , entre los mayores y menores de 0,1 ha producido diferencias significativas.

En cuanto a las estrategias de estimación, nuestros resultados muestran bastantes coincidencias con los de Liu (2009), que, partiendo del modelo de Reys y otros (1982) y complementándolo con las estrategias específicas del trabajo de Levine (1982), elabora un nuevo sistema de tres estrategias: $a$ ) estrategias basadas en la imitación mental del algoritmo escrito, que coinciden con la estrategia de algoritmo simplificado y la imitación del algoritmo escrito; b) estrategias del tipo "redondear y multiplicar", semejantes a la estrategia de primeros dígitos, y c) estrategias alternativas al cálculo escrito, que implican la propiedad distributiva o la factorización, que pueden relacionarse con nuestra estrategia de algoritmo alternativo.

El ámbito de la estimación se ha revelado como ideal para el estudio del error al permitir matices muy ricos, que no se dan en otras áreas de las matemáticas, como la distinción teórica entre razonabilidad, imprecisión, error y otros términos afines. Esta distinción teórica ha tenido su correspondencia en el modelo desarrollado para el análisis de errores, al encontrar categorías de imprecisiones (IA), otras de errores (como los de traducción To y TD) y otras, como la operación con datos diferentes de los propuestos (DI), que no cabe considerarla como categoría de error.

Para el futuro, consideramos prioritarios los trabajos de desarrollo curricular que incorporen la estimación y el sentido numérico en la formación de profesores, y en los distintos niveles educativos, siguiendo la línea de las propuestas curriculares recientes (MEC, 2007; NGA Center \& CCsso, 2010), haciendo que el sentido numérico ocupe un papel central y favoreciendo que la estimación extienda su influencia, fuera del ámbito del cálculo y la medida, para aparecer, como proceso matemático transversal, en todas las áreas de las matemáticas.

\section{REFERENCIAS BIBLIOGRÁFICAS}

Alajmi, A. H. (2009). Addressing computational estimation in the Kuwaiti curriculum: Teachers' views. Journal of Mathematics Teacher Education, 12(4), pp. 263-283. http://dx.doi.org/10.1007/s10857-009-9106-3

Alajmi, A. H. y Reys, R. E. (2010). Examining eighth grade Kuwaiti students' recognition and interpretation of reasonable answers. International Journal of Science and Mathematics Education, 8, pp. 117-139. http://dx.doi.org/10.1007/s10763-009-9165-z

Alajmi, A. H. y Reys, R. E. (2007). Reasonable and reasonableness of answers: Kuwaiti middle school teachers' perspectives. Educational Studies in Mathematics, 65, pp. 77-94. http://dx.doi.org/10.1007/s10649-006-9042-4 
Bestgen, B.; Reys, R. E.; Rybolt, J. F. y Wyatt, J. W. (1980). Effectiveness of systematic instruction on attitudes and computational estimation skills of preservice elementary teachers. Journal for Research in Mathematics Education, 11(2), pp. 124-136. http://dx.doi.org/10.2307/748904

Castro, E. (1991). Resolución de problemas aritméticos de comparación multiplicativa. Granada: Departamento de Didáctica de la Matemática, Universidad de Granada.

Castro, E. y Castro, E. (1996). Conocimiento de contenido pedagógico de los estudiantes de magisterio sobre la estructura multiplicativa. En J. Giménez; S. Llinares y V. Sánchez (eds.). El proceso de llegar a ser un profesor de primaria. Cuestiones desde la educación matemática. Granada: Comares, pp. 119-141.

Center For Best Practices y Council of Chief State School Officers (2010). Common core state standards for mathematics. Washington, DC: NGA Center \& CCsso.

Cortés, J.; Backhoff, E. y Organista, J. (2005). Análisis de estrategias de cálculo estimativo en escolares de secundaria considerados buenos estimadores. Revista Mexicana de Investigación Educativa, 10(25), pp. 543-558.

De Castro, C. (2002). Influencia del tipo de número en la estimación en cálculo. Granada: Universidad de Granada.

De Corte, E. y Verschaffel, L. (1996). An empirical test of the impact of primitive intuitive models of operations on solving word problems with a multiplicative structure. Learning and Instruction, 6, pp. 219-243.

http://dx.doi.org/10.1016/0959-4752(96)00004-7

Edwards, A. (1984). Computational estimation for numeracy. Educational Studies in Mathematics, 15(1), pp. 59-73.

http://dx.doi.org/10.1007/BF00380439

Dowker, A. (1992). Computational estimation strategies of professional mathematicians. Journal for Research in Mathematics Education, 23(1), pp. 45-55.

http://dx.doi.org/10.2307/749163

Fung, M. G. y Latulippe, C. L. (2010). Computational estimation. Teaching Children Mathematics, 17(3), pp. 170-176.

Gómez, B. (1995a). Los métodos de cálculo mental en el contexto educativo: un análisis en la formación de profesores. Granada: Comares.

Gómez, B. (1995b). Tipología de los errores en el cálculo mental. Un estudio en el contexto educativo. Enseñanza de las Ciencias, 13(3), pp. 313-325.

Goodman, T. (1991). Computational estimation skills of pre-service elementary teachers. International Journal of Mathematical Education in Science and Technology, 22, pp. 259-272. http://dx.doi.org/10.1080/0020739910220210

Hanson, S. A. y Hogan, T. P. (2000). Computational estimation skill of college students. Journal for Research in Mathematics Education, 31(4), pp. 483-499. http://dx.doi.org/10.2307/749654

Hiebert, J. y Wearne, D. (1986). Procedures over concepts: The acquisition of decimal number knowledge. En J. Hiebert (ed.). Conceptual and procedural knowledge: The case of mathematics. Hillsdale, NJ: Erlbaum, pp. 199-223

Hogan, T. P. y Brezinski, K. (2003). Quantitative estimation: One, two, or three abilities? Mathematical Thinking \& Learning, 5(4), pp. 259-280. http://dx.doi.org/10.1207/S15327833MTL0504_02

LEVInE, D. R. (1980). Computational estimation ability and the use of estimation strategies among college students. Tesis doctoral. New York University. 
LEvine, D. R. (1982). Strategy use and estimation ability of college students. Journal for Research in Mathematics Education, 13(5), pp. 350-359.

http://dx.doi.org/10.2307/749010

Liv, F. (2009). Computational estimation performance on whole-number multiplication by thirdand fifth-grade Chinese students. School Science and Mathematics, 109(6), pp. 325-337. http://dx.doi.org/10.1111/j.1949-8594.2009.tb18102.x

Ministerio de Educación y Ciencia (1991). Real Decreto 1344/1991, de 6 de septiembre, por el que se establece el currículo de la Educación Primaria. BOE, suplemento del n. ${ }^{\circ} 220$, pp. 3-38.

Ministerio de Educación y Ciencia (2007). Orden ECI/2211/2007, de 12 de julio, por la que se establece el currículo y se regula la ordenación de la Educación Primaria. BOE, 173, pp. 3148731566.

National Council of Teachers of Mathematics (1991). Estándares curriculares y de evaluación para la Educación Matemática. Sevilla: SAEM Thales.

National Council of Teachers of Mathematics (2003). Principios y Estándares para la Educación Matemática. Sevilla: SAEM Thales.

National Council of Teachers of Mathematics (2006). Curriculum focal points for prekindergarten through grade 8 mathematics: A quest for coherence. Reston, VA: NCTM.

Pañellas, M. (2006). Model per a categoritzar les bones estratègies en estimació en numeració i càlcul emprades pels infants en acabar l'educació primària. Aloma: Revista de psicologia, ciències de l'educació $i$ de l'esport, 19, pp. 331-360.

Reys, R. E.; Bestgen, B. J.; Rybolt, J. F. y Wyatt, J. W. (1982). Processes used by good computational estimators. Journal for Research in Mathematics Education, 13(3), pp. 183-201. http://dx.doi.org/10.2307/748555

Rico, L. (1995). Errores en el aprendizaje de las matemáticas. En J. Kilpatrick; P. Gómez y L. Rico (eds.). Educación Matemática. Bogotá \& México: Una Empresa Docente \& Grupo Editorial Iberoamérica, pp. 69-108

Rubenstein, R. N. (1985). Computational estimation and related mathematical skills. Journal for Research in Mathematics Education, 16(2), pp. 106-119.

http://dx.doi.org/10.2307/748368

Schoen, H. L. y Zweng, M. J. (1986). Estimation and mental computation: 1986 Yearbook. Reston, VA: NCTM.

Segovia, I. y Castro, E. (2009). Computational and measurement estimation: curriculum foundations and research carried out at the University of Granada, Mathematics Didactics Department. Electronic Journal of Research in Educational Psychology, 17, vol. 7(1), pp. 499-536.

Segovia, I.; Castro, E.; Castro, E. y Rico, L. (1989). Estimación en cálculo y medida. Madrid: Síntesis.

Segovia, I. y De Castro, C. (2007). La investigación en estimación en cálculo. En E. Castro y J. L. Lupiáñez (eds.). Investigaciones en Educación Matemática: Pensamiento Numérico. Libro homenaje a Jorge Cazares Solórzano. Granada: Editorial Universidad de Granada, pp. 213-236

Siegler, R. S. y Booth, J. L. (2005). Development of numerical estimation: A review. En J. I. D. Campbell (ed.). Handbook of mathematical cognition. Nueva York: Psychology Press, pp. 197-212.

Sowder, J. T. (1988). Mental computation and number comparison: Their roles in the development of number sense and computational estimation. En J. Hiebert y M. Behr (eds.). Number concepts and operations in the middle grades. Reston, va: NCTM, pp. 182-197

Star, J. R. y Rittle-Johnson, B. (2009). It pays to compare: An experimental study on computational estimation. Journal of Experimental Child Psychology, 102(4), pp. 408-26.

http://dx.doi.org/10.1016/j.jecp.2008.11.004 
Star, J. R.; Rittle-Johnson, B.; Lynch, K. y Perova, N. (2009). The role of prior knowledge and comparison in the development of strategy flexibility: The case of computational estimation. ZDMThe International Journal on Mathematics Education, 41, pp. 569-579. http://dx.doi.org/10.1007/s11858-009-0181-9

Volkova, T. N. (2006). Characterizing preservice teachers' thinking in computational estimation with regard to whole numbers, fractions, decimals, and percents. Tesis doctoral, Illinois State University.

YANG, D.; Reys, R. E. y Reys, B. J. (2009). Number sense strategies used by pre-service teachers in Taiwan. International Journal of Science and Mathematics Education, 7, pp. 383-403.

http://dx.doi.org/10.1007/s10763-007-9124-5 


\title{
Multiplicative computational estimation with decimal numbers
}

\author{
Carlos de Castro Hernández \\ Universidad Autónoma de Madrid \\ carlos.decastro@uam.es \\ Enrique Castro Martínez, Isidoro Segovia Álex \\ Universidad de Granada \\ ecastro@ugr.es, isegovia@ugr.es
}

Computational estimation is a basic skill, useful in solving arithmetic problems. In this paper, we study the relative difficulty of estimation tasks depending on the type of operation and number. After that, we analyze computational estimation strategies that student teachers use to estimate the results of multiplicative computations with decimal numbers and, finally, we develop a model for the classification of errors in computational estimation.

We have used a mixed methodology, with two complementary parts: In the quantitative study, with a repeated measures factorial design, we have constructed, administered and analyzed a computational estimation test. In the qualitative part of the study, we have developed the analysis of strategies and errors through a semi-structured interview.

Participants in the study were 131 student teachers: 108 from La Salle, a private center in the Universidad Autónoma de Madrid, and the remaining 23 were students of the Faculty of Education at the Universidad de Granada. Some of the students (52) in the private center were instructed in computational estimation for a period of 10 hours.

A computational estimation test was administered to all the student teachers. Fifty five percent of the 3144 estimates were classified as inaccurate (with an error greater than 30\%). By contrast, 1414 responses (45\%) have had an acceptable degree of accuracy (error equal to or less than 30\%). Through an analysis of variance, we have obtained that both operation type and number type have had a significant effect on dependent variable. Tasks with decimals less than 0.1 are more difficult than others; tasks with decimals less than 1, more difficult than those with natural or decimals greater than 1; and B divisions (with dividend less than divisor) are more difficult than A divisions (with dividend larger than divisor).

In the analysis of computational estimation strategies, we have found four basic strategies: first digits, substitution, fractions, and simplified algorithm. First digits strategy consists in the reduction of the estimation process to the use of number facts, with the leading digits of each number, and determining the order of magnitude of the result. There is usually a reformulation process using an approximation skill, like rounding or truncation, but, in the case of division, the strategy is sometimes performed without reformulation. The strategy of substitution occurs when replacing dividend and divisor by two numbers with the relation of multiple and divisor. In fractions strategy, a decimal number is substituted by a fraction, which affects the phase of calculation and the adjustment of the decimal point. Finally, the alternative algorithm strategy consists in decomposing the dividend as a product of two factors, dividing approximately one factor by the divisor, and multiplying the result by the other factor.

In the error analysis, we have detected errors in the phases of interpretation, execution, and evaluation. In the interpretation phase, we have found substitution errors (incorrect application of an approximation skill), inaccuracies in the approximation skill (an approximation skill, like rounding, applied correctly but with an error greater than 30\%), estimates performed with the data or the operation different from those proposed, and translation errors (substitutions of $\mathrm{a} / \mathrm{b} \div \mathrm{n}$ or $\mathrm{n} \div a / b$ by $(\mathrm{a} \times \mathrm{n}$ ) $\div \mathrm{b}$, or changes in the role of dividend and divisor in a division. In the execution phase, we have found errors in the imitation of the written algorithm, in retrieving number facts, in counting zeros and decimal places to determine the positional value, in the omission of zeros in the adjustment of place value, in a non-placement of the decimal point, in the coordination of the approximation skill with the operation of the decimal point, in a non-appropriate recuperation of a multiplication or division by a power of ten, in operating the decimal point in division as in multiplication, in determining the place value of the first digit of the quotient, or the mistake of starting a division with " 0.0 " instead of " 0 .". Finally, we have called "error of assessment" an explicit replacement of a correct estimation process (which concludes with a reasonable estimation), with an incorrect estimation process that includes an error (due to an incorrect assessment of the estimation process). In summary, $68.5 \%$ of the errors were detected in operations with numbers less than 1 or less than 0.1 , and $60.8 \%$ in the placement of the decimal point. The error (specific of division B) to start a division with " 0.0 " instead of " 0 ." was the most common ( $16.2 \%$ of the errors).

We conclude that the greatest difficulty in computational estimation cannot be found in estimation processes, but in handling decimal numbers (especially those less than 1), in divisions with a dividend less than divisor, and in the adjustment of positional value. It has proved to be important the distinction between divisions A and B. The divisions of type B, as well as being significantly more difficult, have produced specific computational errors. 